\title{
High-resolution UVES/VLT spectra of white dwarfs observed for the ESO SN la progenitor survey
}

\section{DB and DBA stars ${ }^{\star}, \star \star$}

\author{
B. Voss ${ }^{1}$, D. Koester ${ }^{2}$, R. Napiwotzki ${ }^{3}$, N. Christlieb ${ }^{4}$, and D. Reimers ${ }^{4}$ \\ 1 Astronomisches Rechen-Institut, Zentrum für Astronomie der Universität Heidelberg, Mönchhofstr. 12-14, 69120 Heidelberg, \\ Germany \\ e-mail: voss@ari.uni-heidelberg.de \\ 2 Institut für Theoretische Physik und Astrophysik, Universität Kiel, Leibnizstraße 15, 24098 Kiel, Germany \\ 3 Centre for Astrophysics Research, University of Hertfordshire, College Lane, Hatfield AL10 9AB, UK \\ ${ }^{4}$ Hamburger Sternwarte, Gojenbergsweg 112, 21029 Hamburg, Germany
}

Received 13 February 2007 / Accepted 30 May 2007

\section{ABSTRACT}

\begin{abstract}
Context. We present a detailed spectroscopic analysis of the stars with helium-dominated spectra in the ESO Supernova Ia Progenitor Survey (SPY).

Aims. Atmospheric parameters, masses, and abundances of trace hydrogen are determined and discussed in the context of spectral evolution of white dwarfs.

Methods. The spectra are compared with theoretical model atmospheres using a $\chi^{2}$ fitting technique, leading to determinations of effective temperature, surface gravity, and hydrogen abundance.

Results. Our final sample contains 71 objects, of which 6 are new detections and 14 are reclassified from DB to DBA because of the presence of $\mathrm{H}$ lines. One is a cool DO with weak He II lines, 2 are composite DB+dM. 55\% of the DB sample show hydrogen and are thus DBA, a significantly higher fraction than found before.

Conclusions. The large incidence of DBA, and the derived total hydrogen masses are compatible with the scenario that DBs "reappear" around $30000 \mathrm{~K}$ from the DB gap by mixing and diluting a thin hydrogen layer of the order of $10^{-15} M_{\odot}$. This hydrogen mass is then during the evolution continuously increased by interstellar accretion. There are indications that the accretion rate increases smoothly with age or decreasing temperature, a trend which continuous even below the current low temperature limit (Dufour 2006). A remaining mystery is the low accretion rate of $\mathrm{H}$ compared to that of Ca observed in the DBZA, but a stellar wind extending down to the lowest temperatures with decreasing strength might be part of the solution.
\end{abstract}

Key words. stars: white dwarfs - methods: observational - stars: fundamental parameters - stars: atmospheres

\section{Introduction}

About $20 \%$ of all white dwarfs (WD) have atmospheres in which helium is the main constituent. Such hydrogen-deficient stars are believed to form when a cooling stellar remnant experiences a very late helium-shell flash and goes through a born-again episode, i.e., a last thermal pulse, in which almost all residual hydrogen is burnt (see, e.g., Althaus et al. 2005, and references therein).

As these stars advance on the WD cooling sequence, they can initially be observed at temperatures down to approximately $45000 \mathrm{~K}$ as hot DO stars with spectra dominated by He II lines, and subsequently as DB stars whose spectra show only He I, at temperatures that are mostly below $30000 \mathrm{~K}$. Finally, when helium becomes spectroscopically invisible below effective temperatures around $10000 \mathrm{~K}$, the helium atmospheres display either a featureless DC-type spectrum, or a spectrum dominated by the features of trace constituents, i.e., dredged-up carbon leading

* Based on data obtained at the Paranal Observatory of the European Southern Observatory for programmes 165.H-0588 and 167.D-0407.

$\star \star$ Appendix A is only available in electronic form at http://www. aanda.org to DQ spectra or accreted metals leading to a DZ-type spectrum. Besides these main types, DB spectra which show traces of hydrogen or metals are known, classified as DBA or DBZ stars.

While the properties of the hydrogen-atmosphere DA white dwarfs as a group have been closely investigated in numerous studies, the DBs received less attention. They are less numerous than the DAs and thus it is more difficult to assemble data sets that allow meaningful statistical analyses. The most comprehensive study of DB stars so far was that of a sample of $80 \mathrm{DBs}$, carried out by Beauchamp et al. (1996).

Since Beauchamp's study, the number of known DB stars was not significantly increased until two surveys, the Sloan Digital Sky Survey (SDSS, York et al. 2000) and the Supernova Ia Progenitor Survey (SPY, Napiwotzki et al. 2003) collected new samples of DB spectra. The SPY sample contains high-resolution spectra of about $60 \mathrm{DB}$ white dwarfs. These data allow to determine well-constrained parameters of a large number of DB stars for first time since Beauchamp's work. The SDSS spectra, on the other hand, are of medium resolution, but much more numerous. Thus, the SDSS detected a large number of new DBs. The latest version of the catalog of WDs in the SDSS (Eisenstein et al. 2006a), based on the SDSS data release 
4 (DR4), contains 713 DB stars. About 560 of these stars are ordinary DBs which only exhibit He I in their spectra. The second largest subgroup is that of DBA stars with weak Balmer lines in the otherwise He I-dominated spectrum; about 90 such stars are found in the SDSS sample. Many of these newly detected DBs are faint, which makes detailed spectroscopic studies difficult. Nevertheless, the almost tenfold increase of the number of DB stars has allowed the first statistically meaningful analysis of some key questions connected to the evolution of DB stars:

As a helium-atmosphere WD cools, it encounters two temperature regions of special interest, the "DB gap" between temperatures of $45000 \mathrm{~K}$ and $30000 \mathrm{~K}$, and later the instability region of the V777 Her variables near temperatures of $25000 \mathrm{~K}$. The DB gap region has been enigmatic for some time since no DB stars were found in this range of temperatures, raising questions about the very nature of helium-atmosphere white dwarfs. However before the SDSS, only few cool DO and hot DB stars that constrained the gap were known and thus the reality of the gap was not well established. Eisenstein et al. (2006b) found several hot DB stars from the SDSS within the DB gap, some of which exhibit weak He II lines and are thus situated in the higher-temperature part of the DB gap. The gap has however not been closed by their discovery because they also find that the number density of DB stars in the gap is still lower by a factor of about 2.5 than what would be expected from the density of cooler DB stars.

As a possible explanation, it has been speculated that cooling DA stars might change into DBs, provided that their hydrogen layer is very thin, on the order of $M_{\mathrm{H}} \sim 10^{-15} M_{\odot}$. In this case, the superficial hydrogen would be convectively mixed into the more massive helium layer when the atmosphere cools to temperatures lower than $30000 \mathrm{~K}$ and the helium layer becomes convective (see Eisenstein et al. 2006b, and references therein). Some peculiar objects of mixed helium/hydrogen atmosphere compositions, the DAB and some of the DBA stars, might be objects which are currently undergoing such a transition and could therefore provide insight into the viability of this scenario.

The majority of cool DBA stars however can more easily be explained as DB stars that are accreting hydrogen from the ISM. They were long found to comprise about $20 \%$ of the DB stars, a fraction that was first estimated by Shipman et al. (1987) and later confirmed, e.g., by Beauchamp et al. (1996). Similarly, 22\% of all DB stars in the catalogue of McCook \& Sion (1999) are classified as DBA, and in the recent SDSS DR4 sample, the fraction is about $13 \%$. It is clear that the fraction of DBA among the DB stars is a question of the observational hydrogen detection limits.

It has long been expected by some authors that in fact all DBs accrete small traces of hydrogen and would thus appear as DBA if only small enough traces of hydrogen could be detected. Most studies so far, including the SDSS, could only detect hydrogen abundances, relative to helium, larger than a few times $10^{-5}$. These abundance measurements were mostly based on the appearance of $\mathrm{H} \gamma$ and $\mathrm{H} \beta$ in the spectra. Lower abundances can be identified from the Ly $\alpha$ line in UV data (e.g. Provencal et al. 2000), but this method is more involved and not easily applied to large samples. Hunter et al. (2001) demonstrated that also optical spectra allow a measurement of lower hydrogen abundances if they cover the $\mathrm{H} \alpha$ line. In their sample of $24 \mathrm{DB}$ objects, the DBA fraction is raised to about $30 \%$. With observations of a better $S / N$ and higher spectral resolution, this fraction was expected to be even larger, with the expectation that probably hydrogen might be identified in a majority of the DB stars.
Fontaine et al. (2005) proposed that stellar winds of WD stars might prevent hydrogen accretion at temperatures in excess of $20000 \mathrm{~K}$, thus enabling the conversion from DB to DBA stars only at lower temperatures. Observations with increased sensitivity that allow to detect increasingly lower $\mathrm{H}$ masses in DB stars should allow to test this effect and to provide new insight into the evolution of DBA stars.

The high resolution WD spectra that were obtained by SPY contain the largest sample to date of high-quality DB spectra and it should thus be possible to determine lower hydrogen abundances than was possible before from optical data.

The SPY data will also be helpful to investigate the question if invisibly small amounts of trace hydrogen are present in hot DB stars with temperatures above $20000 \mathrm{~K}$. This question is of importance because even such small traces of hydrogen significantly affect the shape of the He I lines and thus complicate the determination of temperatures from the spectra. Beauchamp et al. (1999) compared temperature estimates based on pure He atmospheres to estimates including effects of invisibly small hydrogen contaminations, and found a shift of up to $3000 \mathrm{~K}$ in temperature. The temperatures of hot DBs are therefore not well constrained so far. An improvement of these temperature estimations would greatly benefit the analysis of the V777 Her instability strip.

\section{The SPY data}

SPY is a radial velocity survey that was conducted to test the double degenerate channel of the formation of supernovae Ia. About 800 white dwarfs were observed in the course of the survey, assembling a large collection of high-quality white dwarf spectra. Most of the DB stars among the SPY objects were selected as targets based on the WD catalog (McCook \& Sion 1999) and from a study of DBs in the Hamburg ESO Survey by Christlieb et al. (1997). A more detailed discussion of the target selection for the SPY observations is given in Napiwotzki et al. (2001, 2007).

The SPY spectra were employed for a number of studies beyond the original scope of SPY, of which the work of Koester et al. (2001), hereafter "Paper I", is most relevant to our analysis. They have derived temperatures and gravities from a preliminary sample of about 200 SPY stars, including 13 DB stars.

Since they have already given a detailed description of the data properties, we only briefly summarize the observations and data reduction:

The spectra were obtained with UVES, a high resolution echelle spectrograph at the ESO VLT telescope. UVES was used in a dichroic mode, resulting in small gaps, $\approx 80 \AA$ wide, at $4580 \AA$ and $5640 \AA$ in the final merged spectrum. The spectral resolution at $\mathrm{H} \alpha$ is $R=18500$ or better, and the $S / N$ per binned pixel $(0.05 \AA)$ is $S / N=15$ or higher.

The spectra were reduced with the ESO pipeline for UVES, including the merging of the echelle orders and the wavelength calibration. Paper I found that the quality of these automatically extracted spectra is very good, except for a quasi-periodic wavelike pattern that occurs in some of the spectra. This has since been largely removed by additional processing by collaborators of the SPY project at the University of Erlangen-Nürnberg. A detailed description of the reduction procedure applied to the spectra analyzed here will be published in Napiwotzki et al. (2007). Some artifacts remain in the data, but they do not significantly affect the spectral analysis. 
For our analysis, the data have been rebinned to a step size of $0.1 \AA$. This is in contrast to the analysis of Paper I which used a binning of $1 \AA$. Therefore, we include the Paper I subsample in our analysis, now with the smaller step size. Further details about the observational setup and the data reduction can be found in Napiwotzki et al. (2003) and in Paper I.

\section{Analysis method}

\subsection{Model atmosphere fits}

We fit the data with model spectra from six different grids of helium atmosphere models with different hydrogen abundances, from $\mathrm{H} / \mathrm{He}=10^{-2}$ down to $\mathrm{H} / \mathrm{He}=10^{-7}$, and with a grid of pure He atmosphere models. The grids cover a range of temperatures from $10000 \mathrm{~K}$ to $50000 \mathrm{~K}$ and logarithms of the surface gravities from 7.0 to 9.0. The input physics of the models are described in Finley et al. (1997), Homeier et al. (1998), Koester \& Wolff (2000), and Koester et al. (2005a). We use a $\chi^{2}$ minimization fitting routine that is based on the Levenberg-Marquardt algorithm (Press et al. 1992) to derive the best fitting effective temperature and surface gravity for each spectrum. Some more details on the fitting process can be found in Homeier et al. (1998).

The SPY spectra are not reliably flux calibrated, and therefore we do not use their continuum slopes for the fitting but only the profiles of the helium lines. We determine a reference continuum level at each wavelength to which the models are scaled; this reference level is derived by interpolating the flux levels measured next to the He I lines.

The helium lines reach their maximum strength around $T_{\text {eff }}=20000 \mathrm{~K}$, and therefore two solutions for the fit of a spectrum are normally possible, one on either side of the line strength maximum. We employed two $T_{\text {eff }}$ starting values for the fitting, $15000 \mathrm{~K}$ and $25000 \mathrm{~K}$, to cover all possible solutions.

For most objects it is not difficult to pick the correct solution; if the temperature difference of both solutions is large, the line profiles are different enough to allow an easy decision for the best fit. Furthermore, for many objects there is only one solution, either because both fits converge to a single solution, or because the fit from the hot starting value converges to exceedingly high values of the temperature. For the remaining few objects, a careful study of the fit quality was necessary to determine which solution is to be preferred; for some objects the hydrogen abundance or the presence of pulsations provided additional constraints.

There are two stars in our sample that show composite spectra. The red part of the spectra was not used in the fits of these $\mathrm{DB}+\mathrm{dM}$ binaries; only the He I lines at short wavelengths of $\lambda<4500 \AA$ were fitted, where the flux of the late-type companion is negligible.

Below effective temperatures of approximately 15500 to $16500 \mathrm{~K}$ - depending on surface gravity - neutral He van der Waals broadening becomes more and more important for the line shapes. Since our original model grids included only Stark broadening, we have calculated a second grid up to $18000 \mathrm{~K}$ which includes Stark broadening in a simplified form and van der Waals broadening combined. This greatly improved the agreement with the observed line profiles and strengths. Because the fit was not perfect, and also because in general the dependence of line profiles on surface gravity is smaller for the cooler DBs, we have decided to keep $\log g$ fixed at 8.0, in order to get a more robust estimate of the effective temperature. A fit in which $\log g$ is allowed to vary would converge to very high gravity values.

There are six objects in our sample whose spectra only show a strongly broadened $5876 \AA$ line, or only this line together with about equally strong $\mathrm{H} \alpha$. These are very cool objects with helium-rich atmospheres; they are related to peculiar stars like, e.g., HS 0146+1847 (Koester et al. 2005a), which are dominated by helium but appear hydrogen-rich because of their very low temperature. Since only one or two lines are available in these spectra, no meaningful fit would be possible and thus we estimate the temperature and the hydrogen abundance from the equivalent widths of $\mathrm{He}$ I $5876 \AA$, and, if present, $\mathrm{H} \alpha$, assuming a fixed $\log g=8.0$.

\subsection{Determination of $\mathrm{H} / \mathrm{He}$}

We obtain seven different fit results for each observed spectrum, one fit result per assumed hydrogen abundance. If a visual inspection of the observed spectrum does not reveal the presence of $\mathrm{H} \alpha$, we adopt the pure helium atmosphere solution.

Otherwise, if $\mathrm{H} \alpha$ is unambiguously present, as shown in Figs. A.1 through A.3, we measure the equivalent width of the line in the observed spectrum as well as in each of the six model spectra. Then, we interpolate the model equivalent widths $E W$, model temperatures $T_{\text {eff }}$, and model gravities $\log g$ as functions of the assumed hydrogen abundance $\mathrm{H} / \mathrm{He}: E W(\mathrm{H} / \mathrm{He})$, $T_{\text {eff }}(\mathrm{H} / \mathrm{He})$, and $\log g(\mathrm{H} / \mathrm{He})$. With the observed equivalent width $E W_{\text {obs }}$, we can determine $\mathrm{H} / \mathrm{He}_{\text {obs }}$ from the inversion of $E W(\mathrm{H} / \mathrm{He})$. We then adopt $T_{\text {eff }}\left(\mathrm{H} / \mathrm{He}_{\mathrm{obs}}\right)$ and $\log g\left(\mathrm{H} / \mathrm{He} \mathrm{obs}_{\mathrm{os}}\right)$ as the temperature and gravity of the object.

For most stars, the $\mathrm{H} \alpha$ equivalent width does not rise monotonously over the whole range of modeled abundances. Instead, it mostly rises monotonously up to $\mathrm{H} / \mathrm{He}=10^{-3}$ and decreases again for $\mathrm{H} / \mathrm{He}=10^{-2}$. The reason for this effect is that a fit of the $\mathrm{H} / \mathrm{He}=10^{-2}$ models to the data results in a high temperature, for which the equivalent width is small in spite of the high abundance. A given equivalent width thus often allows two solutions for the $\mathrm{H}$ abundance. For all but three of our objects the low-abundance solution proves to be the better fit of the data. For the objects for which instead the high abundance solution is preferred, the fit quality difference of both solutions is small. Therefore it has to be noted that for these objects a second solution of almost identical quality exists.

\subsection{Stellar masses and hydrogen mass fractions}

The masses were determined from $T_{\text {eff }}$ and $\log g$, and the evolutionary mass-radius relations of Wood (1995) for "thin" layers, appropriate for DB white dwarfs.

The depth of the convection zones in DB white dwarfs for given mass and effective temperature were calculated with our stellar structure code. This code was originally developed for the calculation of finite-temperature mass-radius relations and equilibrium models for pulsation studies (Koester 1978; Dziembowski \& Koester 1981). This code is not fully evolutionary, but solves the static stellar structure equations; The energy equation is replaced by setting $l(r) \propto m(r)$. This is a sufficiently accurate approximation, since in the outer layers it results in $l \approx L=$ const. and in the interior the temperature becomes nearly constant because of the high heat conduction of the degenerate electrons. The input physics - equation of state and opacities have been updated and the outer boundary values are determined from accurate DB atmospheric models. The parameterization of 
Table 1. Properties and parameters of the DB stars without visible hydrogen. Two lines of data are given for the hot objects $\left(T_{\text {eff }}>20000 \mathrm{~K}\right)$; the second line contains the result of a fit with $\mathrm{H} / \mathrm{He}=10^{-5}$ models.

\begin{tabular}{|c|c|c|c|c|c|c|c|c|}
\hline Object & RA & Dec & mag & Alias & $T_{\text {eff }}[\mathrm{K}]$ & $\log g$ & $M_{*}\left[M_{\odot}\right]$ & Comments \\
\hline WD 0119-004 & $01: 21: 48.27$ & $-00: 10: 54.4$ & $V=16.0$ & G 271-047A, LP 587-44, SDSS & 16542 & 8.0 & & 4 \\
\hline WD 0127-311 & 01:29:56.11 & $-30: 55: 09.6$ & $V=14.51$ & HE 0127-3110, GD 1363 & 11002 & 8.0 & & only He I $5876 \AA^{3}$ \\
\hline МСТ0149-2518 & $01: 51: 59.62$ & $-25: 03: 15.1$ & $B=15.91$ & PHL 1201 & 16834 & 7.940 & 0.557 & \\
\hline WD 0158-160 & 02:00:56.69 & $-15: 46: 09.7$ & $B=14.38$ & G 272-B2B, MCT & $\begin{array}{l}25518 \\
25259\end{array}$ & $\begin{array}{l}7.875 \\
7.891\end{array}$ & 0.542 & 1 \\
\hline WD 0203-181 & $02: 05: 23.98$ & $-17: 53: 25.8$ & $V=16.0$ & HE 0203-1807, G 272-152 & 10757 & 8.0 & & only He I $5876 \AA^{3}$ \\
\hline WD 0249-052 & $02: 52: 15.55$ & $-05: 02: 32.5$ & $B=16.1$ & HE 0245-0514, KUV & 17908 & 7.992 & 0.589 & \\
\hline HE $0308-5635$ & 03:09:47.84 & $-56: 23: 49.6$ & $B=14.02$ & WD 0308-565, BPM 17088 & $\begin{array}{l}22849 \\
22822\end{array}$ & $\begin{array}{l}8.060 \\
8.081\end{array}$ & 0.639 & 1 \\
\hline WD $0349+015$ & 03:51:51.65 & $+01: 39: 47.6$ & $B=16.3$ & KUV $03493+0131$ & 18741 & 7.889 & 0.535 & \\
\hline HE 0417-5357 & 04:19:10.04 & $-53: 50: 45.7$ & $B=15.12$ & BPM 17731 & 18733 & 7.942 & 0.563 & \\
\hline HE 0420-4748 & $04: 22: 11.38$ & $-47: 41: 42.3$ & $B=14.7$ & & $\begin{array}{l}27288 \\
27245\end{array}$ & $\begin{array}{l}7.808 \\
7.809\end{array}$ & 0.512 & 1 \\
\hline HE 0423-1434 & 04:25:51.72 & $-14: 27: 52.0$ & $B=16.21$ & & 16904 & 7.794 & 0.481 & new object \\
\hline HE 0429-1651 & $04: 32: 13.81$ & $-16: 45: 08.5$ & $B=15.82$ & & 15659 & 7.659 & & $\mathrm{DB}+\mathrm{dM}$; new object \\
\hline WD 0615-591 & $06: 16: 14.48$ & $-59: 12: 28.1$ & $V=14.09$ & L 182-61, BPM 18164 & 16714 & 8.017 & 0.600 & \\
\hline WD 0845-188 & $08: 47: 29.52$ & $-18: 59: 50.7$ & $V=15.55$ & LP 786-06, L 0748-70 & 17566 & 7.969 & 0.575 & \\
\hline WD 0900+142 & 09:03:31.31 & $+14: 00: 49.2$ & $B=16.48$ & PG 0900+142 & 15678 & 8.0 & & 4 \\
\hline WD 1004-178 & 10:07:07.91 & $-18: 05: 25.8$ & $V=15.6$ & EC $10047-1750$ & 16357 & 7.700 & & $\mathrm{DB}+\mathrm{dM}$ \\
\hline WD 1046-017 & $10: 48: 32.65$ & $-02: 01: 11.3$ & $V=15.81$ & GD 124, GR 387 & 15138 & 8.0 & & 4 \\
\hline WD 1144-084 & $11: 46: 54.07$ & $-08: 45: 48.5$ & $B=15.95$ & PG 1144-085 & 16468 & 8.0 & & 4 \\
\hline WD 1252-289 & $12: 54: 55.30$ & $-29: 11: 54.6$ & $V=15.85$ & EC 12522-2855 & 21029 & 7.97 & 0.595 & \\
\hline WD 1326-037 & $13: 29: 16.37$ & $-03: 58: 51.8$ & $V=15.6$ & PG 1326-037 & $\begin{array}{l}22645 \\
22192\end{array}$ & $\begin{array}{l}7.999 \\
7.995\end{array}$ & 0.603 & $\begin{array}{l}2 \\
1\end{array}$ \\
\hline WD $1336+123$ & 13:39:13.63 & $+12: 08: 29.6$ & $B=13.9$ & LP 498-26 & 16779 & 8.0 & & 4 \\
\hline WD $1428-125$ & $14: 31: 39.64$ & $-12: 48: 55.5$ & $V=15.98$ & EC 14289-1235 & $\begin{array}{l}21586 \\
21657\end{array}$ & $\begin{array}{l}8.167 \\
8.179\end{array}$ & 0.701 & 1 \\
\hline WD $1445+152$ & $14: 48: 14.37$ & $+15: 04: 49.6$ & $B=15.55$ & PG $1445+153$ & $\begin{array}{l}23234 \\
24063\end{array}$ & $\begin{array}{l}8.012 \\
7.968\end{array}$ & 0.611 & 1 \\
\hline WD $1542+182$ & $15: 44: 19.46$ & $+18: 06: 43.6$ & $V=14.72$ & GD 190, EG 193 & $\begin{array}{l}23186 \\
22902\end{array}$ & $\begin{array}{l}7.966 \\
7.965\end{array}$ & 0.585 & 1 \\
\hline WD 1612-111 & $16: 15: 23.96$ & $-11: 18: 29.5$ & $V=15.53$ & GD 198, EG 194 & $\begin{array}{l}22143 \\
22338\end{array}$ & $\begin{array}{l}8.073 \\
8.076\end{array}$ & 0.645 & 1 \\
\hline WD $1654+160$ & $16: 56: 57.56$ & $+15: 56: 26.6$ & $B=16.15$ & PG 1654+160, V 824 Her & $\begin{array}{l}25447 \\
25501\end{array}$ & $\begin{array}{l}7.846 \\
7.839\end{array}$ & 0.527 & $\begin{array}{l}\text { V777 Her variable } \\
1\end{array}$ \\
\hline WD $2129+000$ & $21: 32: 16.39$ & $+00: 15: 13.3$ & $V=14.73$ & LP 638-4, PHL 028 & 14414 & 8.0 & & 4 \\
\hline WD 2144-079 & $21: 47: 37.34$ & $-07: 44: 13.1$ & $V=14.82$ & G 026-031, LTT 8702 & 16518 & 7.895 & 0.532 & DBZ \\
\hline WD 2234+064 & $22: 36: 42.06$ & $+06: 40: 16.4$ & $y=16.26$ & PG 2234+064, GR 907 & $\begin{array}{l}20890 \\
19864\end{array}$ & $\begin{array}{l}8.035 \\
8.028\end{array}$ & 0.620 & 1 \\
\hline WD 2354+159 & $23: 56: 34.74$ & $+16: 15: 39.8$ & $B=15.78$ & PG 2354+159 & $\begin{array}{l}24428 \\
22822\end{array}$ & $\begin{array}{l}8.180 \\
8.218\end{array}$ & 0.713 & $\begin{array}{l}\text { DBZ } \\
1\end{array}$ \\
\hline WD 2354-305 & $23: 56: 37.07$ & $-30: 16: 25.4$ & $B=16.26$ & & 18006 & 7.883 & 0.530 & \\
\hline
\end{tabular}

${ }^{1}$ Fit with $\mathrm{H} / \mathrm{He}=10^{-5}$ models to check the influence of possible admixtures of invisibly small amounts of hydrogen.

${ }^{2}$ Beauchamp et al. (1999) list WD 1326-037 as a DBA; however no H is visible in the SPY spectra. According to Bergeron (2007, priv. comm.), the Beauchamp data show no hydrogen features and thus this object appears as a DBA in their paper due to a mis-classification.

${ }^{3}$ Temperatures were determined from the equivalent widths of He I $5876 \AA$. The surface gravity was assumed to be $\log g=8.0$.

${ }^{4}$ Fitted with the alternate set of models with van der Waals line broadening. The surface gravity was kept fixed at $\log g=8.0$ for these fits.

the mixing length version is ML2, $\alpha=0.6$ (see e.g. Fontaine et al. 1981; Tassoul et al. 1990; or Koester et al. 1994 for an explanation of nomenclature). The mass-radius relation predicted by these models is in reasonable agreement with more sophisticated calculations (e.g. Wood 1995).

This code predicts the total mass in the convection zone as a function of the mass and effective temperature of the star. By multiplying this with the hydrogen abundance we obtain the total hydrogen mass within the convection zone in solar masses.

\section{Results}

The properties and fit results of the DB stars without visible hydrogen in their atmosphere are listed in Table 1. The data for the DBA stars are shown in Table 2.

\subsection{Uncertainties}

The formal uncertainties from the $\chi^{2}$ fitting routine are mostly low, and normally amount to only a few times $10 \mathrm{~K}$. However, 


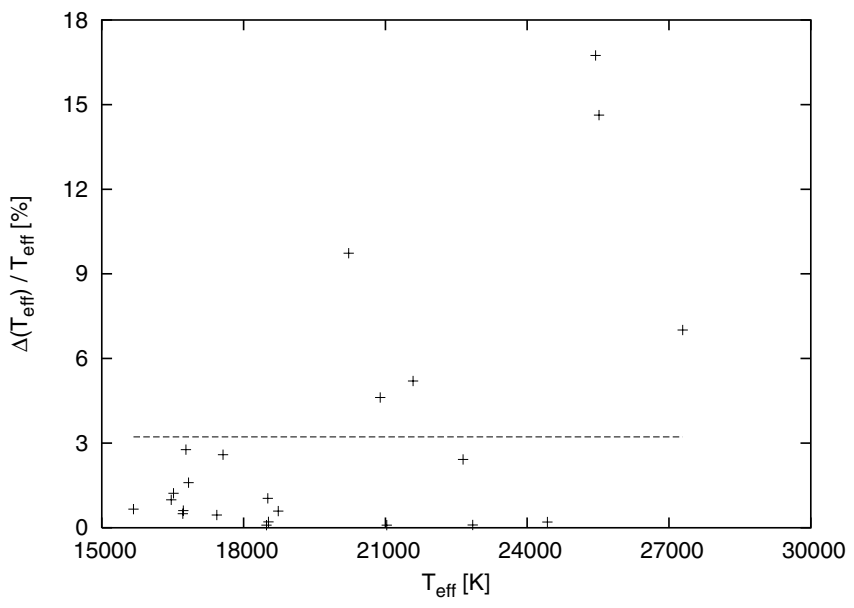

Fig. 1. The relative differences of the temperatures that we derive for two spectra of the same object.

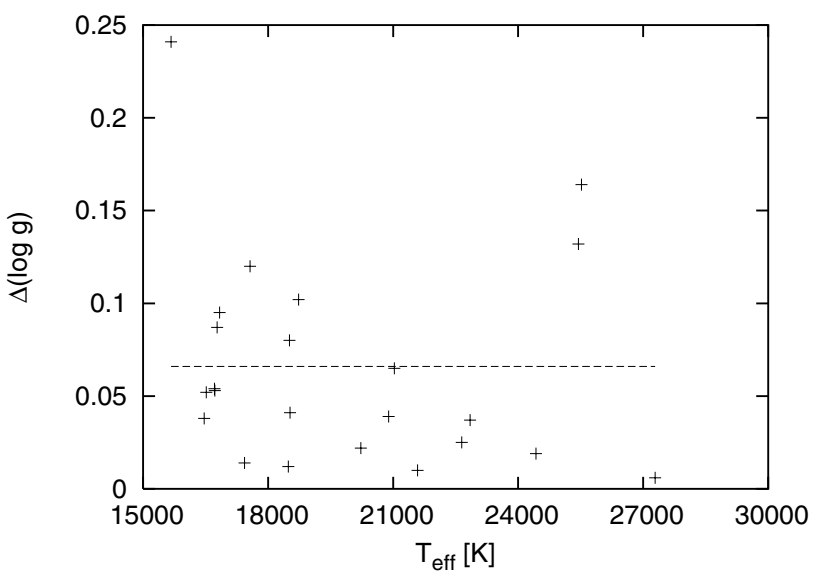

Fig. 2. The same as Fig. 1, for $\log g$.

they do not include systematic errors, which might be introduced by the data reduction, or by the lack of a flux calibration of the data. Thus the true uncertainties of the fit results are generally underestimated by the formal uncertainties. A better estimate can be obtained by comparing the fit results of the two independent SPY spectra of each object, an approach that was earlier used by Liebert et al. 2005 in their study of DA white dwarfs. We have done this for 24 of our objects for which both spectra are not too different in quality and arrive at mean differences of $\left\langle\Delta T_{\text {eff }} / T_{\text {eff }}\right\rangle=3.22 \%$ and $\langle\Delta \log g\rangle=0.066$. The large $\left\langle\Delta T_{\text {eff }}\right\rangle$ is dominated by two outliers at high temperatures. If these are omitted, the results are $\left\langle\Delta T_{\text {eff }} / T_{\text {eff }}\right\rangle=2.03 \%$ and $\langle\Delta \log g\rangle=$ 0.058 . These results are plotted in Figs. 1 and 2 .

For six of the DBA objects we have conducted the full analysis for both spectra and can thus also directly estimate the uncertainties of $\mathrm{H} / \mathrm{He}$ and $M_{\mathrm{H}}$; five of the six objects show very low differences of abundance and hydrogen mass, $\langle\Delta \log (\mathrm{H} / \mathrm{He})\rangle=$ 0.02 and $\left\langle\Delta \log \left(M_{\mathrm{H}}\right)\right\rangle=0.025$, i.e., the total $\mathrm{H}$ masses differ by, on average, $6 \%$. These estimates are of course based on a small number of objects and thus only allow to judge the order of magnitude of the uncertainties.

A comparison to the parameters that other authors derive for common objects looks less satisfying; the differences in temperature between our results and those of Beauchamp et al. (1999), Friedrich et al. (2000), and Castanheira et al. (2006), shown in Fig. 3, are scattered by more than $\pm 10 \%$ and the results of

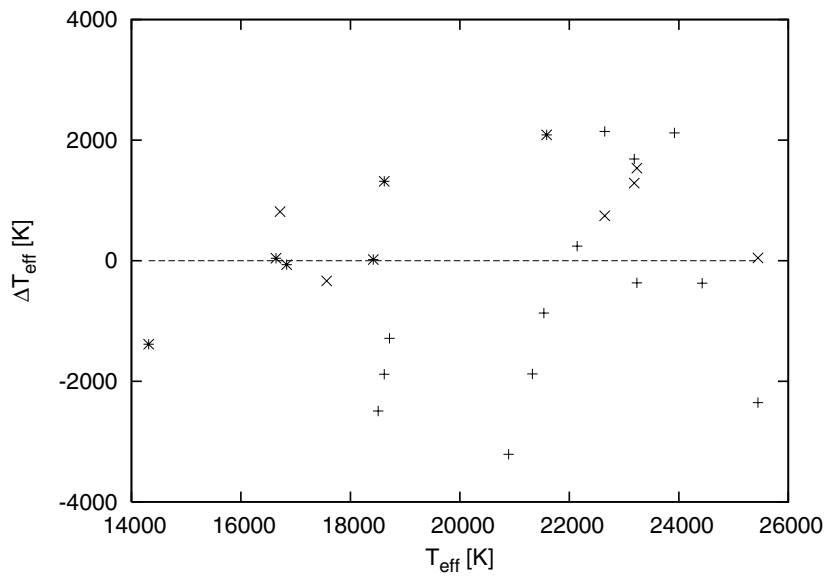

Fig. 3. The differences of the temperatures that we and other authors derive for common objects, plotted against our temperature. The "+"symbols show the comparison to the results of Beauchamp et al. (1999), the " $\times$ "-symbols that to Castanheira et al. (2006) and the asterisks plot the comparison to the values of Friedrich et al. (2000).

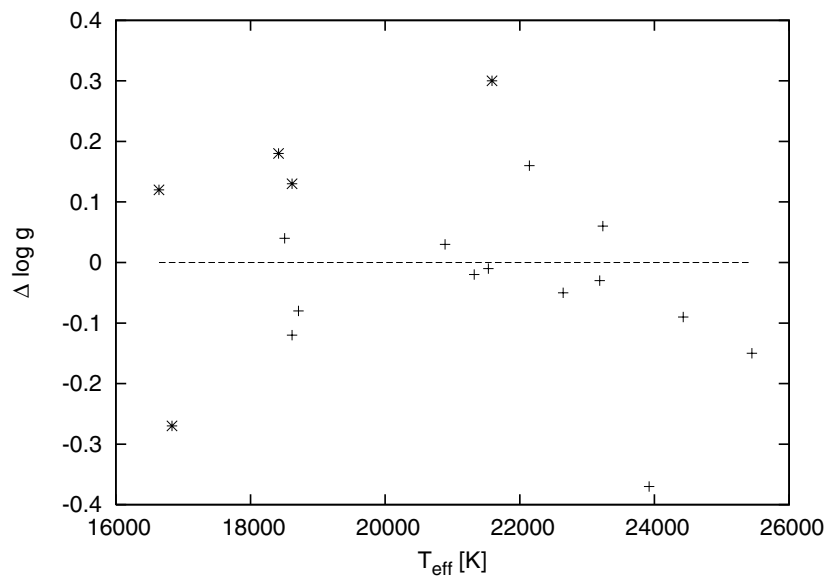

Fig. 4. The same as Fig. 3, for $\log g$.

Beauchamp seem systematically hotter by a few $\%$, or about $1500 \mathrm{~K}$. The surface gravity differences, shown in Fig. 4, scatter by over \pm 0.15 . It has to be noted that Castanheira et al.'s results are derived from UV data, and that they use only pure helium models. Therefore we only compare our results for DB without hydrogen to their data. Beauchamp et al. assumed hydrogen abundances, whereas we can use observed $\mathrm{H}$ lines. They assume $\mathrm{H} / \mathrm{He}=10^{-2}$ for their DBA stars, and certain lower hydrogen abundances corresponding to their detection limits for stars that they do not identify as DBA. The systematic differences of our results to theirs may in part arise because for all of our DBA stars that are in common with Beauchamp's sample, we derive lower abundances than he assumed.

\subsection{Hot DB stars}

Low amounts of hydrogen are spectroscopically invisible in hot atmospheres but can influence the shape of the helium lines through a change of the stratification and line broadening and thus affect the result of a model atmosphere fit. To test this effect we have fitted the DB stars with $T_{\text {eff }}>20000 \mathrm{~K}$ also with models of atmospheres with an admixture of an invisibly small amount of hydrogen, $\mathrm{H} / \mathrm{He}=10^{-5}$. These fit results are also listed in Table 1 . The surface gravities do not differ 


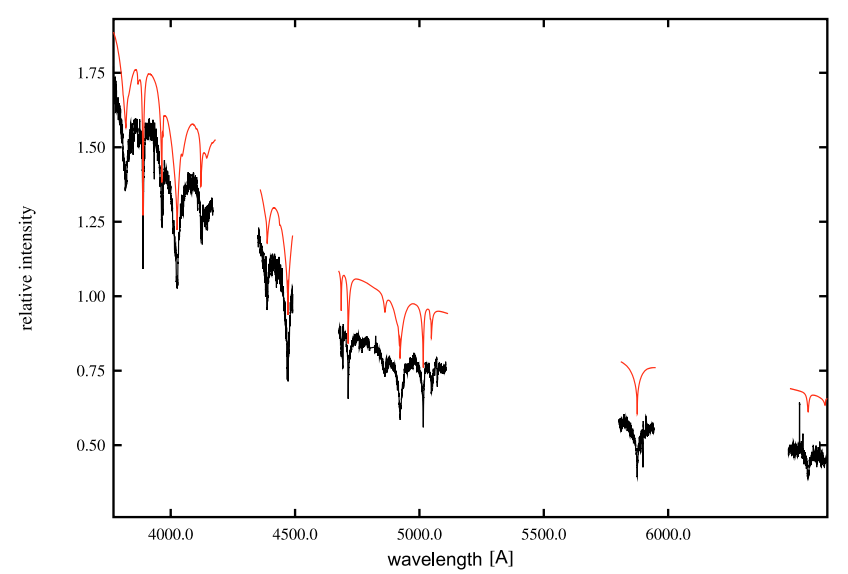

Fig. 5. The fitted part of the spectrum of WD2142-169, shown as a black line, and the fit in red, shifted upwards by a constant amount for clarity.

significantly to those derived from hydrogen-free atmosphere models; the temperatures are on average larger if hydrogen is mixed into the atmosphere. The average temperature difference is $\overline{\Delta T_{\text {eff }}}=231 \mathrm{~K}$, and thus on the order of the uncertainties of the measurements. The unknown hydrogen abundance therefore adds some additional uncertainty to the atmosphere parameters of hot DB stars, but this effect is much less pronounced in our results than in those of Beauchamp et al. (1999) and Castanheira et al. (2006). This is not surprising because the higher resolution of our spectra allows us to use an upper limit of the abundance of spectroscopically invisible that is much lower than that of the earlier investigations, who used upper limits between $\mathrm{H} / \mathrm{He}=$ $10^{-3.5}$ (Castanheira et al.) and $\mathrm{H} / \mathrm{He}=10^{-4}$ (Beauchamp et al.).

Our sample includes only two V777 Her variables, and thus our results do not allow any meaningful statement on the structure of the V777 Her instability strip.

\subsection{Peculiar objects}

\subsubsection{The Cool DO Star WD 2142-169}

The spectrum of WD 2142-169 is dominated by He I lines, but also shows a weak He II $4686 \AA$ line, as well as $\mathrm{H} \alpha, \mathrm{H} \beta$, and $\mathrm{Ca}$ II lines. In a classification determined by the relative strength of the spectral features this star would be a DBO; however in accordance with Eisenstein et al. (2006b) we classify it as a DO rather than a DB or DBO. The presence of He II indicates that the temperature can not be much smaller than $40000 \mathrm{~K}$, but the weakness of the line suggests that the temperature of WD 2142-169 is lower than that of most other DO stars, almost all of which have $T_{\text {eff }}>45000 \mathrm{~K}$.

WD 2142-169 is listed as a DB star of $T_{\text {eff }}=15900 \mathrm{~K}$ in Koester et al. (2005b), and in fact such a fit can be obtained at a moderate fit quality, and with a low hydrogen abundance of $\mathrm{H} / \mathrm{He}=10^{-5}$, but of course such a fit does not reproduce the He II line. The best fit is achieved with a much higher hydrogen abundance, $\mathrm{H} / \mathrm{He}=10^{-2}$, and results in a temperature of $T_{\text {eff }}=39100 \mathrm{~K}$ and a gravity of $\log g=7.74$. This fit is shown in Figs. 5 and 6. Note that NLTE effects, which are important in hotter DOs, disappear below 40000 K (Dreizler \& Werner 1996). The He II line is slightly stronger in the fit than in the spectrum, indicating that the atmosphere is perhaps slightly cooler than the fit result. The Balmer lines are not well reproduced by this fit; they are stronger in the observed spectrum than

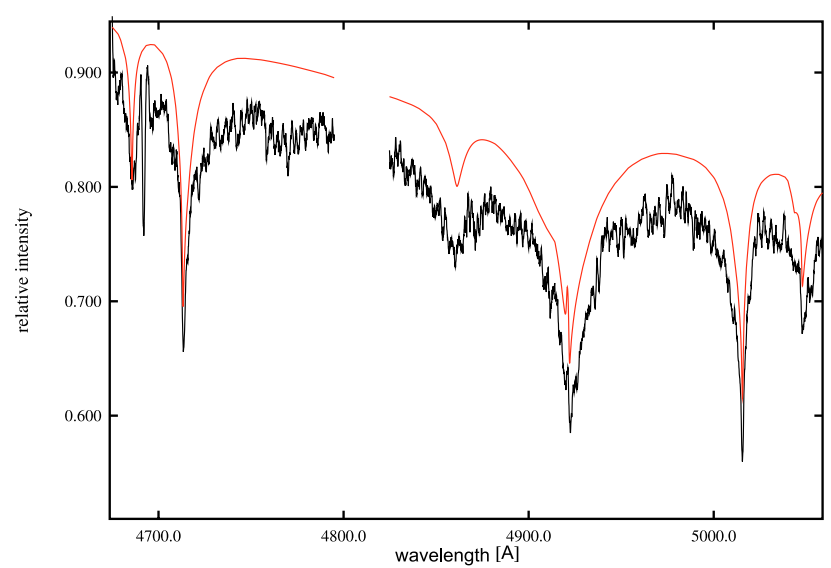

Fig. 6. The part of the spectrum of WD2142-169 that contains the He II $4686 \AA$ and $\mathrm{H} \beta$ lines. The spectrum is shown as a black line, and the fit in red, shifted upward. The feature at $4690 \AA$ is caused by a defect of the CCD that appears in many SPY spectra.

in the fitted model, and thus the $\mathrm{H}$ abundance is probably higher than fitted, $\mathrm{H} / \mathrm{He}>10^{-2}$. A fit with a model of a low hydrogen abundance usually overestimates the temperature. Thus, both the He II line and the hydrogen features point to a true temperature that is even slightly lower than $39000 \mathrm{~K}$.

WD 2142-169 is therefore one of the coolest DO stars that are known and one of very few helium-rich objects that populate the DB gap. Only one similar object is known so far, SDSS J074538.1+312205 (Eisenstein et al. 2006b). Like WD 2142-169, it shows weak He II and Balmer lines in a spectrum that is dominated by He I. Eisenstein et al. derive a temperature of $39800 \pm 2000 \mathrm{~K}$ from the SDSS photometry, however they do not obtain a reliable fit of the objects' spectrum.

\subsubsection{WD 0453-295}

This apparent DAB white dwarf was studied by Wesemael et al. (1994) and found to be a DA+DB binary, since no single star atmosphere model fit is able to reproduce the flux distribution of this object.

The SPY spectra of WD 0453-295 (see Fig. 7) show variable line core shapes, due to the orbital motion of the components. Napiwotzki et al. (2005) found that the core of $\mathrm{H} \alpha$ is split in one of the spectra. Thus the DB component is indeed a DBA or perhaps even a DAB star, whose $\mathrm{H}$ abundance has to be considerable, since the equivalent widths of $\mathrm{H} \alpha$ of both the DA and its companion have to be comparable.

\subsubsection{HE 2149-0516}

The spectrum of HE 2149-0516 shows strong Balmer lines and weaker but strong He I lines. It is a new DAB star. A pure helium (or hydrogen) atmosphere fit does not give a result since the line strengths are too strongly affected by the other constituent of the atmosphere; only a pure hydrogen atmosphere fit that is limited to the $\mathrm{H} \alpha$ line results in a reasonable temperature estimate of $29000 \mathrm{~K}$ to $30000 \mathrm{~K}$, but with a very low fitted gravity of $\log g \approx 6.9$. Alternatively, we find $T_{\text {eff }}=32000 \mathrm{~K}$ if the gravity is kept fixed at $\log g=8.0$. These fits of the line are not good and the results are thus only very rough estimates. Nevertheless, the resulting temperature is similar to those that were derived for most other stars of this class, i.e., near the low-temperature edge of the DB gap. 


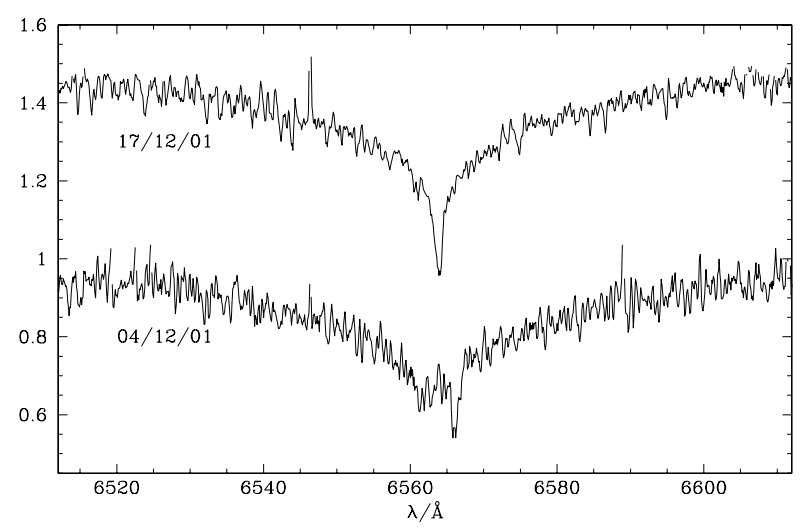

Fig. 7. The H $\alpha$ line in the two SPY spectra of WD 0453-295. The lower spectrum shows two line cores. Taken from Napiwotzki et al. (2005).

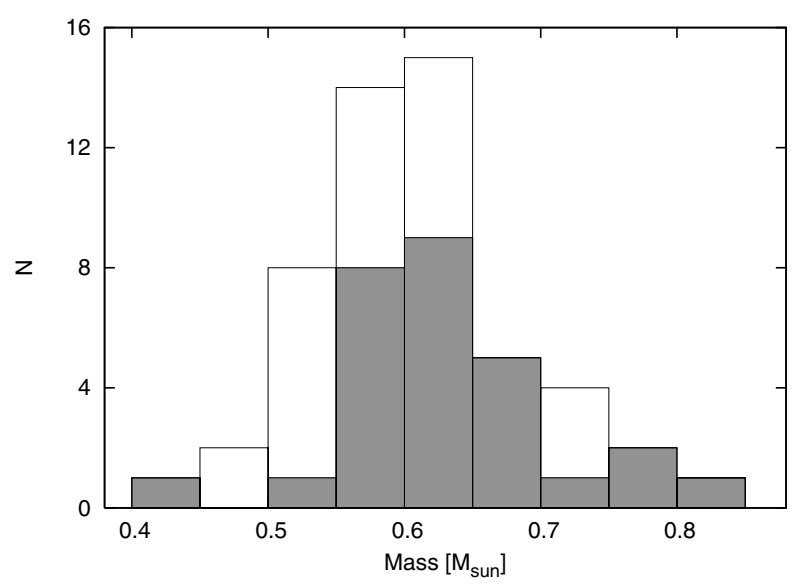

Fig. 8. The mass distribution of the DB stars. The filled part of each bin represents the DBA stars.

\section{4. $D B$ and DBA mass distributions}

Figure 8 shows the mass distribution of the DB and DBA stars. The magnetic DBA WD 0853+163, the cool DO, and the objects for which $\log g$ was kept fixed during the model atmosphere fit are not included in this plot. The overall shape of the distribution is similar to that which is found for the DA stars by, e.g., Liebert et al. (2005), Giovannini et al. (2005), or Madej et al. (2004), as well as for the SPY DA stars (Voss et al. 2007, in preparation). The mean mass of all stars is $0.596 \pm 0.072 M_{\odot}$, and thus very similar to the average mass of the DA stars that has long been found to be about $0.55-0.6 M_{\odot}$. The similarity is especially striking for the subsample of DBs that do not show any hydrogen in their spectra; their mean mass is $0.584 \pm 0.059 M_{\odot}$. The mean mass of the DBA subsample is only very slightly higher, $0.607 \pm$ $0.082 M_{\odot}$. Such a trend towards higher masses in DBA stars was already found by Beauchamp et al. (1996), although much more pronounced: the mean masses for their sample of 13 DBA stars and $41 \mathrm{DB}$ without spectroscopically visible hydrogen are $\langle M\rangle=$ $0.642 M_{\odot}$ for the DBA and $\langle M\rangle=0.567 M_{\odot}$ for the DB. They speculated that the presence of hydrogen could be favored by a larger stellar mass through an increased hydrogen accretion from the interstellar medium, or through a thinner convection zone in which hydrogen dilution would be decreased.

However, the differences that we find between our DB and DBA distributions are not significant. Furthermore, to test Beauchamp et al.'s speculation, we tried to identify any dependency of the hydrogen abundance, the hydrogen mass, or the

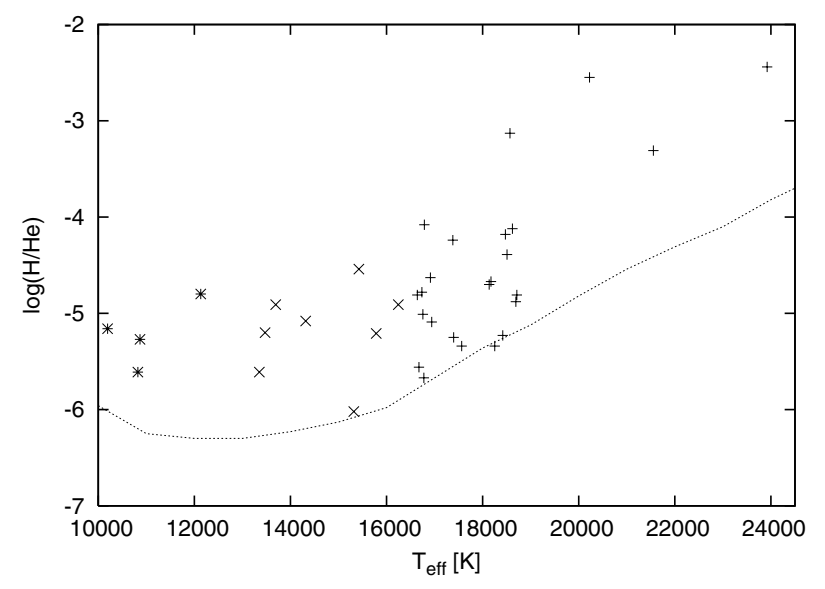

Fig. 9. The hydrogen abundances of the DBA stars as a function of the effective temperature. The tilted crosses show the objects for which the alternate model grids were used and for which the surface gravity was kept fixed during the fit. Asterisks show the stars for which temperatures and abundances were derived from the equivalent line widths of He I $5876 \AA$ and $\mathrm{H} \alpha$, respectively. The dotted line corresponds to the $\mathrm{H} \alpha$ equivalent width detection limit of $300 \mathrm{~m} \AA$.

hydrogen accretion rate on the stellar mass. No such dependency is found, which adds confidence to the assumption that DBAs and DBs are not intrinsically different from each other.

Beauchamp et al. also found that the distribution of the DB masses is narrower than that of the DA stars, only $5 \%$ of their DB stars are placed outside of a mass interval from $0.5 M_{\odot}$ to $0.65 M_{\odot}$. Here, a fraction of $25 \%$ of the DBs are found outside that interval, which is not too different from the $32 \%$ of the SPY DA stars that have masses outside this range.

Finally, the DB mean mass of this analysis is much lower than the unexplainedly high value of $0.77 M_{\odot}$ that was found in Paper I. The difference is probably due to the fact that Paper I used an earlier, less sophisticatedly reduced version of the data. A major improvement was a better correction of the response function of the spectrograph in the blue spectra. The imperfect correction in the previous version was likely responsibe for a significant fraction of the systematic offset. The reason for the high Paper I masses may be not entirely clear, but it is reassuring that we now find a mass distribution that agrees with that of the well studied $80 \%$ of all White Dwarfs, the DA. According to these results, there is no need to assume any distinction between the structures of white dwarfs with hydrogen- or helium-rich atmospheres, or, for that matter, between DB and DBA stars.

\subsection{Hydrogen abundance and total hydrogen mass in the DBA stars}

Figure 9 shows that the observed hydrogen abundances are lower for cooler DBA stars than for the hottest objects. This decrease of the average abundance is not unexpected since the helium convection zone deepens with decreasing temperature, leading to increased dilution of any hydrogen mass originally present.

The lowest observed $\mathrm{H} \alpha$ equivalent widths in our DBA sample are close to $300 \mathrm{~m} \AA$, and therefore we assume this value as the detection limit for hydrogen in our data. The detection limit in terms of abundance vs. temperature is shown by the dotted line in the figure. The observed abundances reach near this limit at temperatures around $18000 \mathrm{~K}$ but are higher for the hottest and coolest objects. This reflects the average quality of our 


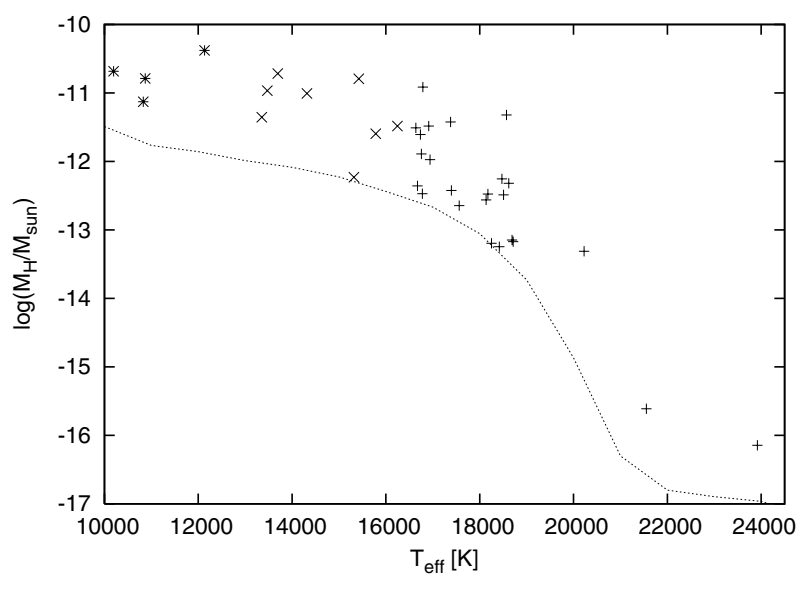

Fig. 10. The hydrogen masses plotted against the effective temperature; the symbols and the dotted line are the same as in Fig. 9.

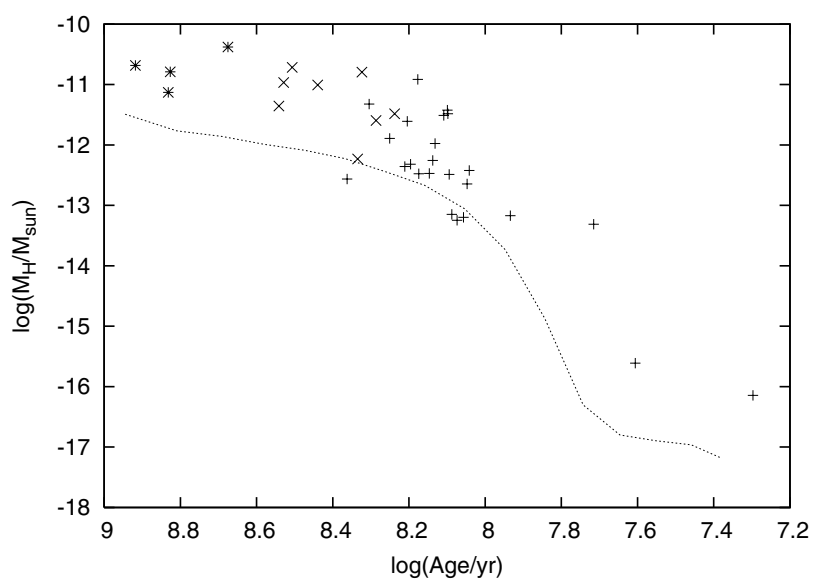

Fig. 11. The hydrogen masses plotted against $\log ($ Age $)$; the symbols are again the same as in Fig. 9. The dotted line depicts the hydrogen detection limit for $\log g=8.0$. This $300 \mathrm{~m} \AA$-limit would be placed at higher ages for stars with $\log g>8.0$.

spectra which is somewhat lower for the hot and cool objects, and better at intermediate temperatures.

A more instructive quantity is the hydrogen mass itself, which is shown in Fig. 10 as a function of temperature and in Fig. 11 as a function of white dwarf age. Since we assumed a constant mass below approx. $16000 \mathrm{~K}$, the qualitative similarity between the two curves is not too surprising. Both of them clearly show that the deduced $\mathrm{H}$ masses increase with the age of the WD. Before interpreting this in a too simplistic way one should note, however, the observational detection limit, which prevents any observation of $\mathrm{H}$ masses below $10^{-12} M_{\odot}$ at the cool end. On the other hand, the absence of larger $\mathrm{H}$ masses at the hot end is explained easily: a WD with $\mathrm{H}$ mass of $10^{-12} M_{\odot}$ at $22000 \mathrm{~K}$ would have a $\mathrm{H} / \mathrm{He}$ number ratio $>1$, and thus look more like a DAB than a DBA.

\section{Discussion and conclusions}

The detection of low hydrogen abundances in such a large number of spectra significantly increases the number of DBA stars among the white dwarfs with helium-rich atmospheres. With a fraction of $55 \%$, the majority of DBs in our sample are DBAs. The increase is not unexpected because it seemed always plausible that decreasing the detection limit would discover even

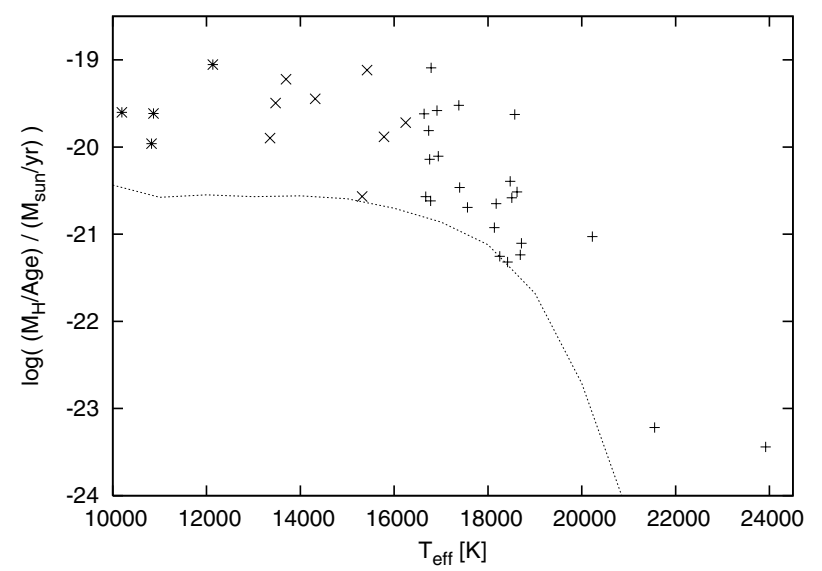

Fig. 12. The hydrogen accretion rate, shown on a logarithmic scale against the effective temperature. The symbols are the same as in the previous figures.

weaker hydrogen lines. There are 6 new objects in our sample which have not been reported before in the literature, and 4 of these are DBAs. In addition to that, 14 stars of our sample cataloged as DBs turn out to be DBAs. One of them is the V777 Her variable WD $1115+158$.

How do these observations fit with our understanding of DB evolution and the DB gap? The evolution of a $0.6 M_{\odot}$ DA in the DB gap and its transformation into a DB by convective mixing has been studied in great detail by MacDonald \& Vennes (1991). Depending on the convection description used, the transition can occur between 25000 and $32000 \mathrm{~K}$, if the hydrogen mass is $10^{-15} M_{\odot}$. For $10^{-14} M_{\odot}$ of H mass these values go down to $11800-18000 \mathrm{~K}$. Since we observe DBA white dwarfs up to $24000 \mathrm{~K}$ in our sample, we assume $\approx 10^{-15} M_{\odot}$ as a reasonable starting value for those DA within the DB gap, which will turn into hot helium-rich stars around $30000 \mathrm{~K}$. Because of the shallow convection zone this leads to the high observed values of $\log \mathrm{H} / \mathrm{He}=-3$ to -2 ; the apparently pure He DBs in this temperature range may have just slightly lower $\mathrm{H}$ masses. Because of the steep increase of the convective mass such small amounts of hydrogen would become unobservable after a short evolution to temperatures below $20000 \mathrm{~K}$. We still find hydrogen in these cooler stars, but now indicating much larger total $\mathrm{H}$ masses of $\geq 10^{-13} M_{\odot}$. We can envisage two scenarios for the origin of this hydrogen: Mixing of a thicker original $\mathrm{H}$ layer, i.e. transformation of a DA into a DB at much lower temperatures than the canonical $30000 \mathrm{~K}$, or interstellar accretion.

In view of the MacDonald \& Vennes (1991) calculations we can discard the first scenario. None of their different convection models allows mixing of $10^{-13} M_{\odot}$ at a temperature higher than $12500 \mathrm{~K}$, whereas such masses are observed already at $18000 \mathrm{~K}$. Assuming a constant accretion rate over the lifetime of the $\mathrm{DB}$, the accretion rates necessary to explain the observations are extremely low: between $10^{-19}$ and $10^{-21} M_{\odot}$ per year (see Figs. 12 and 13). In their important study of the diffusion/accretion scenario for DZ white dwarfs, Dupuis et al. (1993) estimate a rate of $2.7 \times 10^{-22} M_{\odot}$ for a typical WD in the thin, warm phase of the interstellar medium. This is based on the Eddington rate, using just the gravitational cross section of a star in classical mechanics. It is very hard to imagine a lower rate, unless some unknown mechanism completely prevents accretion, but in any case such or even lower rates would not lead to observable hydrogen. On the other hand, a significantly higher rate - continuous or episodic - would lead to $\mathrm{H} / \mathrm{He}$ ratios of 0.01 


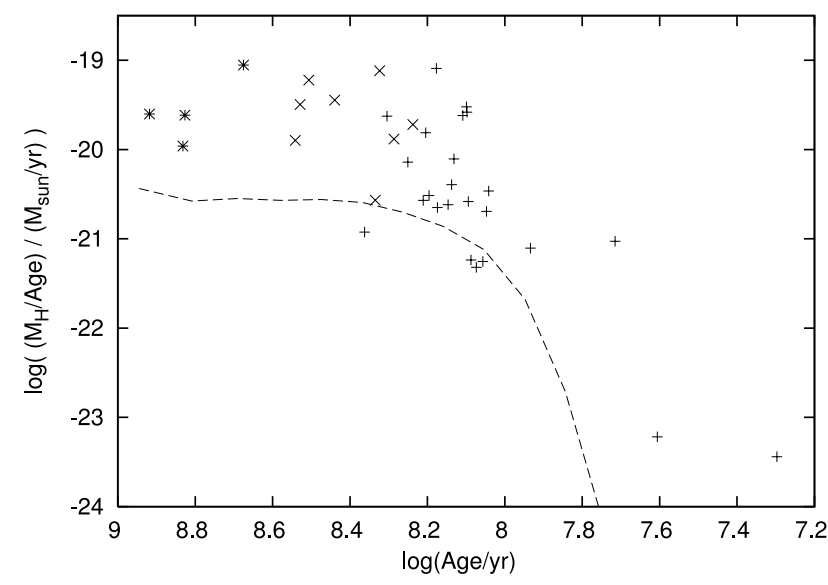

Fig. 13. The hydrogen accretion rate, shown on a logarithmic scale against the age of the star. The symbols are the same as in the previous figures.

to 0.1 at the low temperature end, as observed in HS 0146+1847 (Koester et al. 2005a) and GD362 (Zuckerman et al. 2007). These stars appear at first sight to be normal DAs, and only careful observation and analysis reveals their unusual composition. It is currently completely unknown how many more of these objects might be hidden among the "normal" DAs. Overall, our results thus strongly support the idea that small but varying amounts of hydrogen are accreted over their lifetimes by all DB white dwarfs.

The recent study by Dufour et al. (2007) of DZ white dwarfs provides a very interesting continuation of our results towards lower temperatures. In the overlap region between 10000 and $12000 \mathrm{~K}$, our results for the $\mathrm{H} / \mathrm{He}$ ratios, total $\mathrm{H}$ masses, and necessary accretion rates are in very good agreement. With some precaution because of the selection effects, we can extend their findings of the decreasing $\mathrm{H}$ accretion rate with effective temperature much beyond their limit of $12000 \mathrm{~K}$ up to $24000 \mathrm{~K}$. This may have some relevance for one of the remaining mysteries, which is strongly re-emphasized by Dufour et al. (2007): In those cases, where metal traces are detected in addition to $\mathrm{H}$ (the DBZ, or DBZA), the accretion rate for $\mathrm{H}$ compared to e.g. $\mathrm{Ca}$ is orders of magnitude below that expected from solar abundances in the accreted matter. If there is some kind of screening mechanism involved, which prevents or hinders the accretion of hydrogen, it seems to increase its efficiency in a rather smooth way towards high temperatures. This would argue against the propeller mechanism (Wesemael \& Truran 1982), which is most often invoked as a possible explanation. The only other possibility we are aware of is the existence of a weak stellar wind in hot DBs, which dies down with decreasing temperature. In their wind model to explain the carbon abundance in DBs, Fontaine et al. (2005) assumed a linear relationship between wind strength and age, and that it dies at $20000 \mathrm{~K}$. Whether such a very weak wind (comparable in absolute value to our derived accretion rates) exists down to very much lower temperatures, and whether it can explain the difference in the $\mathrm{Ca} / \mathrm{H}$ accretion rates remains to be studied.
Acknowledgements. B.V. and D.K. acknowledge support by the Deutsche Forschungsgemeinschaft (DFG) under project numbers KO738/21-1, KO738/22-1, and KO738/23-1.

\section{References}

Althaus, L. G., Serenelli, A. M., Panei, J. A., et al. 2005, A\&A, 435, 631 Beauchamp, A., Wesemael, F., Bergeron, P., Liebert, J., \& Saffer, R. A. 1996, ASPC, 96, 295

Beauchamp, A., Wesemael, F., Bergeron, P., et al. 1999, ApJ, 516, 887

Castanheira, B. G., Kepler, S. O., Handler, G., \& Koester, D. 2006, A\&A, 450, 331

Christlieb, N., Karl, C., Wisotzki, L., Reimers, D., \& Koester, D. 2007, A\&A, submitted

Dreizler, S., \& Werner, K. 1996, A\&A, 314, 217

Dufour, P. 2006, Ph.D. Thesis, Montreal

Dufour, P., Bergeron, P., Liebert, J., et al. 2007, ApJ, in press

Dupuis, J., Fotaine, G., Pelletier, C., \& Wesemael, F. 1993, ApJS, 84, 73

Dziembowski, W., \& Koester, D. 1981, A\&A, 97, 16

Eisenstein, D. J., Liebert, J., Harris, H. C., et al. 2006a, ApJS, 167, 40

Eisenstein, D. J., Liebert, J., Koester, D., et al. 2006b, AJ, 132, 676

Finley, D. S., Koester, D., \& Basri, G. 1997, ApJ, 488, 375

Fontaine, G., Villeneuve, B., \& Wilson, J. 1981, AJ, 243, 550

Fontaine, G., \& Brassard, P. 2005, in 14th European Workshop on white dwarfs, ed. D. Koester, \& S. Moehler, ASP Conf. Ser., 334, 49

Friedrich, S., Koester, D., Christlieb, N., Reimers, D., \& Wisotzki, L. 2000, A\&A, 363, 1040

Giovannini, O., da Costa, A. F. M., Castanheira, B. G., \& Kepler, S. O. 2005, in 14th European Workshop on White Dwarfs, ed. D. Koester, \& S. Moehler, ASP Conf. Ser., 334, 89

Homeier, D., Koester, D., Hagen, H.-J., et al. 1998, A\&A, 338, 563

Hunter, C., Wesemael, F., Saffer, R. A., Bergeron, P., \& Beauchamp, A. 2001, in ASP Conf. Proc., 226, ed. J. L. Provencal, H. L. Shipman, J. MacDonald, \& S. Goodchild, 153

Koester, D. 1978, A\&A, 64, 289

Koester, D., \& Wolff, B. 2000, A\&A, 357, 587

Koester, D., Allard, N. F. \& Vauclair, G. 1994, A\&A, 291, L9

Koester, D., Napiwotzki, R., Christlieb, N., et al. 2001, A\&A, 378, 556

Koester, D., Napiwotzki, R., Voss, B., Homeier, D., \& Reimers, D. 2005a, A\&A, 439,317

Koester, D., Rollenhagen, K., Napiwotzki, R., et al. 2005b, A\&A, 432, 1025

Liebert, J., Bergeron, P., \& Holberg, J. B. 2005, ApJS, 156, 47

MacDonald, J., \& Vennes, S. 1991, ApJ, 371, 719

Madej, J., Nalezyty, M., \& Althaus, L. G. 2004, A\&A, 419, 5

McCook, G. P., \& Sion, E. M. 1999, ApJS, 121, 1

Napiwotzki, R., Christlieb, N., Drechsel, H., et al. 2001, AN, 322, 411

Napiwotzki, R., Christlieb, N., Drechsel, H., et al. 2003, ESO Msngr., 112, 25

Napiwotzki, R., Karl, C. A., Nelemans, G., et al. 2005, in 14th European Workshop on White Dwarfs, ed. D. Koester, \& S. Moehler, ASP Conf. Ser., 334, 89

Napiwotzki, R., et al. 2007, in preparation

Press, W. H., Teukolsky, S. A., Vetterling, W. T., \& Flannery, B. P. 1992, Numerical Recipes, second edition, The Press Syndicate of the University of Cambridge, Cambridge, New York, Melbourne

Provencal, J. L., Shipman, H. L., Thejll, P., \& Vennes, S. 2000, ApJ, 542, 1041

Shipman, H. L., Liebert, J., \& Green, R. F. 1987, ApJ, 315, 239

Tassoul, M., Fontaine, G., \& Winget, D. E. 1990, ApJ, 358, 196

Voss, B., Koester, D., Napiwotzki, R., et al. 2007, in preparation

Wesemael, F., \& Truran, J. W. 1982, ApJ, 260, 807

Wesemael, F., Bergeron, P., Lamontagne, R., et al. 1994, ApJ, 429, 369

Wood, M. A. 1995, in White Dwarfs, Proceedings of the 9th European Workshop on White Dwarfs held at Kiel, Germany, Lecture Notes in Physics, ed. D. Koester, \& K. Werner (Berlin, Heidelberg, New York: Springer-Verlag), 443, 41

York, D. G., Adelman, J., Anderson, J. E. Jr., et al. 2000, AJ, 120, 1579

Zuckerman, B., et al. 2007, in preparation 
B. Voss et al.: High-resolution spectra observed for SPY. II., Online Material p 1

\section{Online Material}


B. Voss et al.: High-resolution spectra observed for SPY. II., Online Material p 2

\section{Appendix A: Wavelength region around $\mathrm{H} \alpha$ in the DBAs}
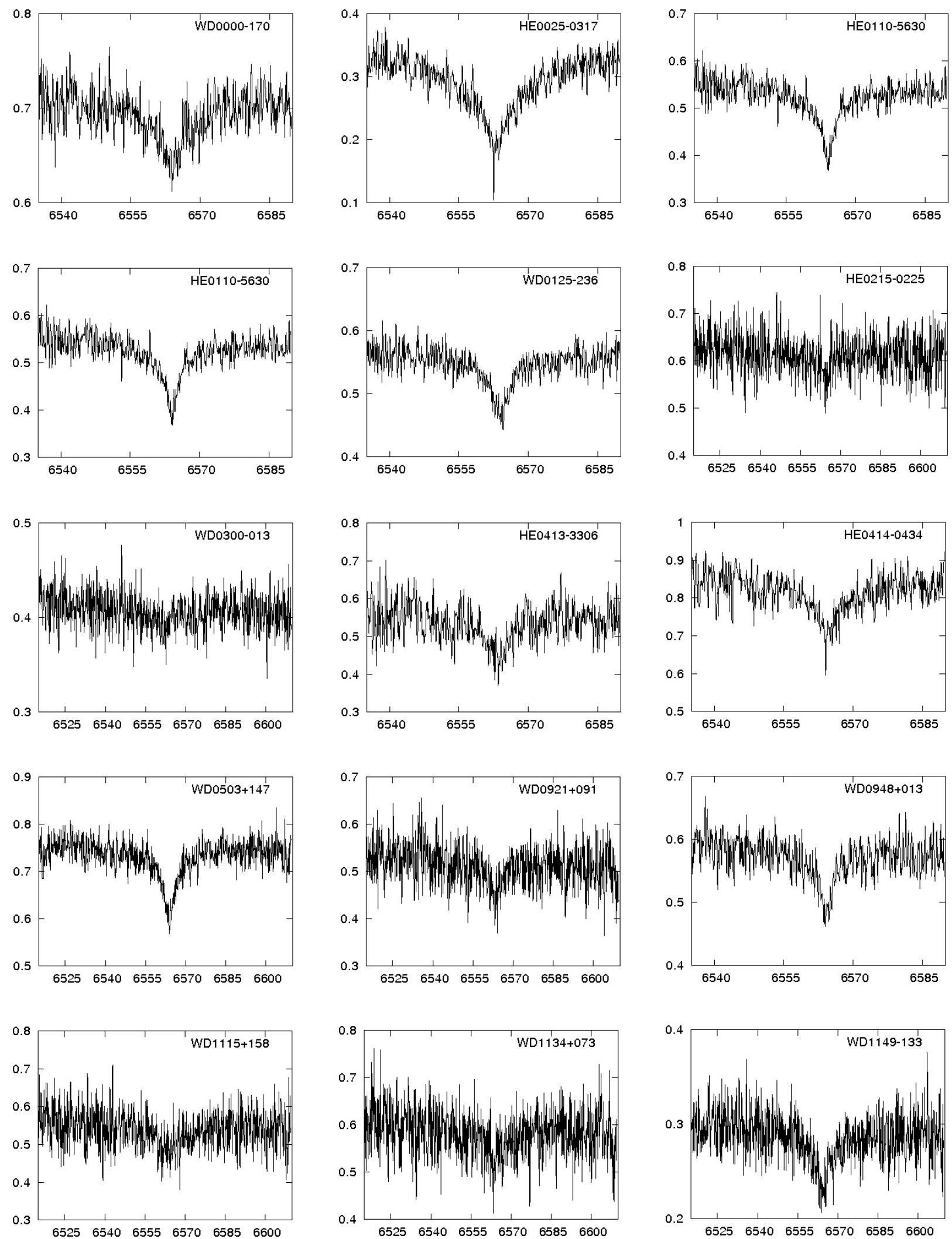

Fig. A.1. The spectral regions around $\mathrm{H} \alpha$ for all DBA stars, continued in Figs. A.2 and A.3. The intensity is given in arbitrary units; the wavelengths are in $\AA$. Note that the wavelength range is not the same in all plots. 
B. Voss et al.: High-resolution spectra observed for SPY. II., Online Material p 3
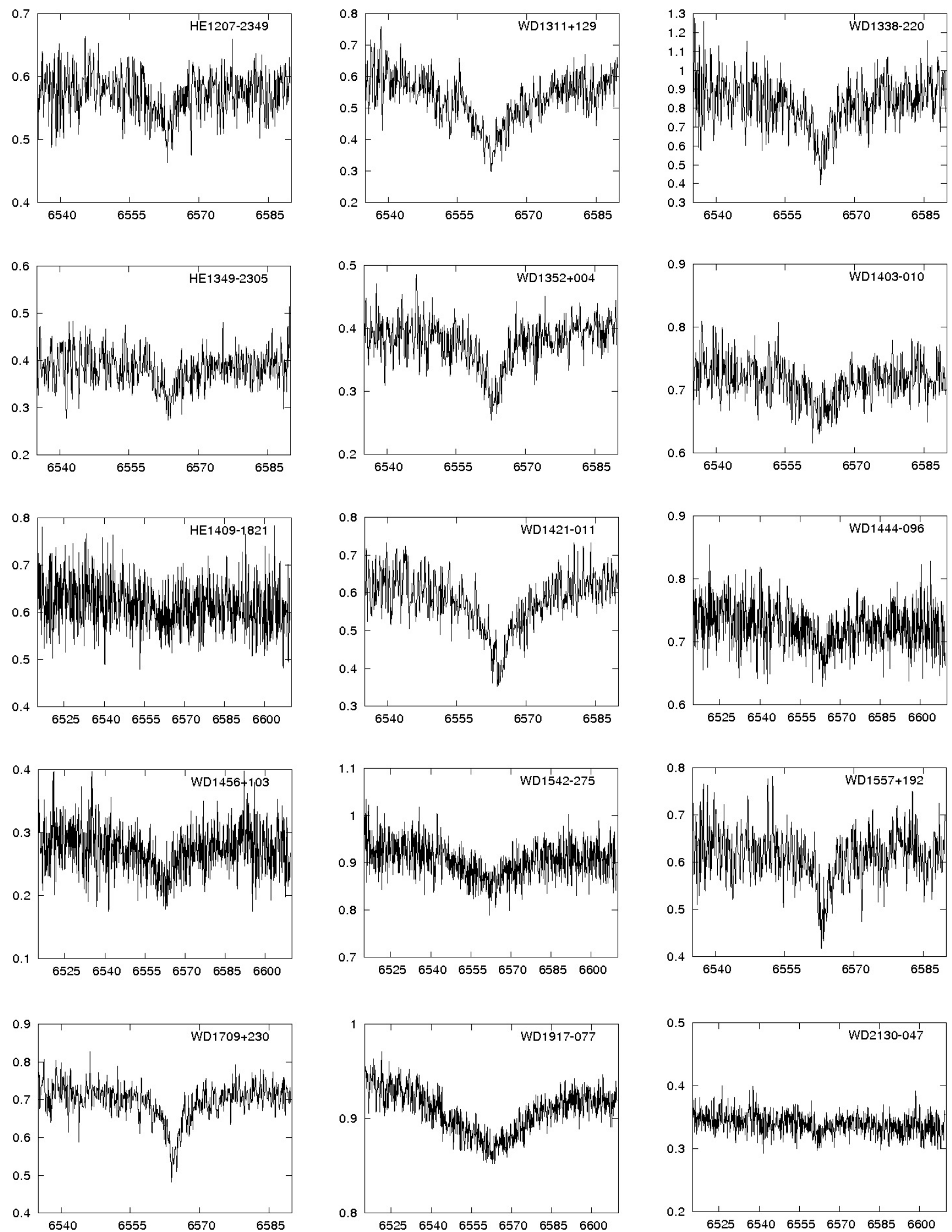

Fig. A.2. The spectral regions around $\mathrm{H} \alpha$ for all DBA stars, continued from Fig. A.1. 
B. Voss et al.: High-resolution spectra observed for SPY. II., Online Material p 4
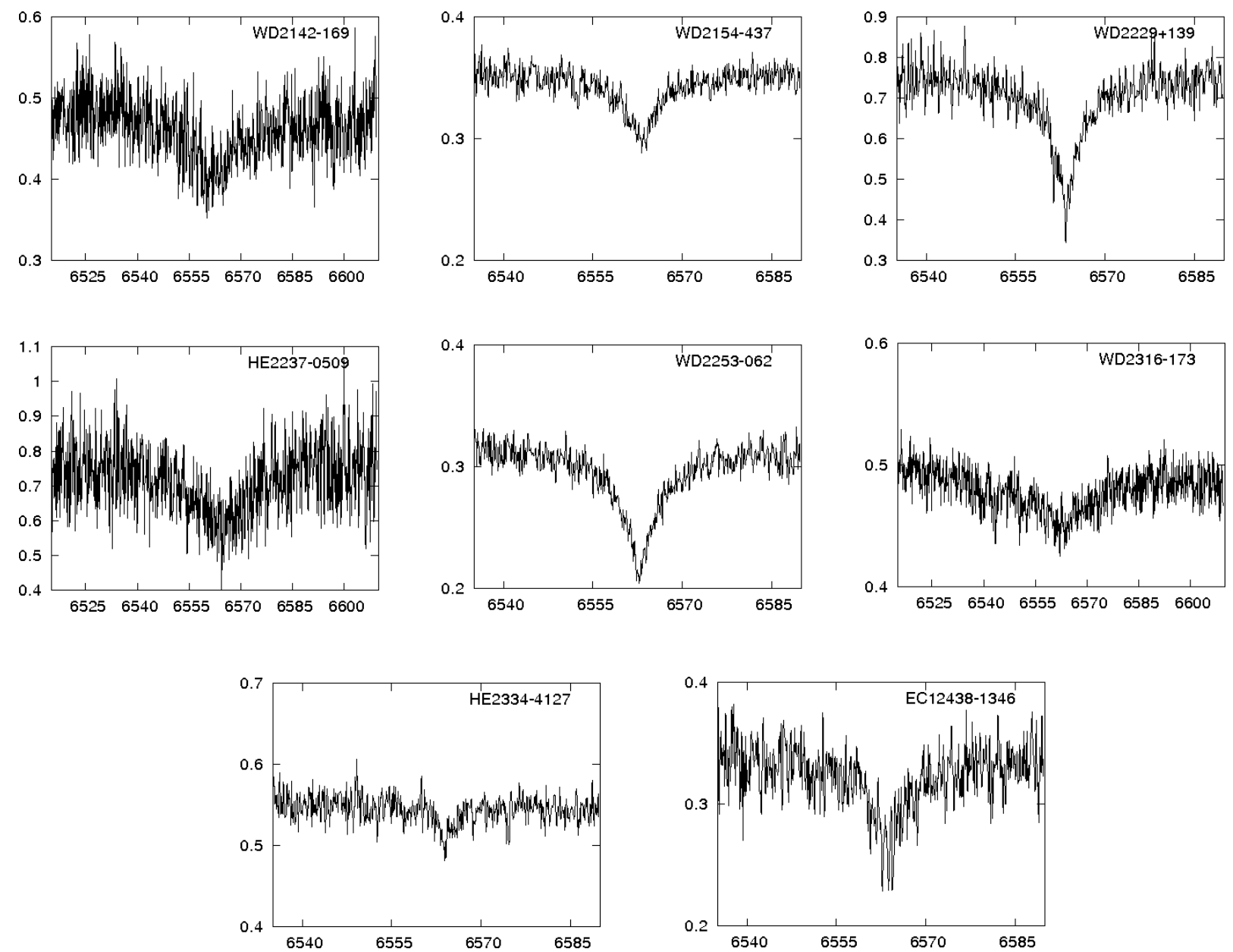

Fig. A.3. The spectral regions around $\mathrm{H} \alpha$ for all DBA stars, continued from Figs. A.1 and A.2. 